University of Nebraska - Lincoln

DigitalCommons@University of Nebraska - Lincoln

John R. Hardy Papers

Research Papers in Physics and Astronomy

8-15-1989

\title{
Calculation of Dielectric Susceptibility for Complex Ionic Systems: Application to a Predicted Superlattice
}

\author{
J. L. Feldman \\ Naval Research Laboratory, Washington, D. C. \\ L. L. Boyer \\ Naval Research Laboratory, Washington, D. C. \\ P. J. Edwardson \\ Naval Research Laboratory, Washington, D. C.
}

Follow this and additional works at: https://digitalcommons.unl.edu/physicshardy

Part of the Physics Commons

Feldman, J. L.; Boyer, L. L.; and Edwardson, P. J., "Calculation of Dielectric Susceptibility for Complex Ionic Systems: Application to a Predicted Superlattice" (1989). John R. Hardy Papers. 32.

https://digitalcommons.unl.edu/physicshardy/32

This Article is brought to you for free and open access by the Research Papers in Physics and Astronomy at DigitalCommons@University of Nebraska - Lincoln. It has been accepted for inclusion in John R. Hardy Papers by an authorized administrator of DigitalCommons@University of Nebraska - Lincoln. 


\title{
Calculation of dielectric susceptibility for complex ionic systems: Application to a predicted superlattice
}

\author{
J. L. Feldman, L. L. Boyer, and P. J. Edwardson \\ Condensed Matter Physics Branch, Condensed Matter and Radiation Science Division, Naval Research Laboratory, \\ Washington, D.C. 20375-5000 \\ J. R. Hardy \\ University of Nebraska-Lincoln, Lincoln, Nebraska 68588-0111 \\ (Received 9 May 1988; revised manuscript received 23 March 1989)
}

\begin{abstract}
The Gordon-Kim theory of interionic interactions between closed-shell ions, which has had considerable success for existing alkali halide systems, has been applied to the determination of the structure, phonon dispersion, and room-temperature dielectric properties of a proposed superlattice, $\mathrm{RbF}(\mathrm{NaCl})_{2}$, of alkali halides. The dielectric properties of such a hypothetical material can have an unusually strong low-frequency dependence because of the presence of Brillouin-zonefolded "infrared"-active modes. In evaluating the standard expressions for the dielectric properties, in the rigid-ion approximation, we make use of standard cubic and quartic anharmonic terms in addition to the instantaneous-phonon cubic anharmonic term, which is needed because of the lack of a center of inversion. Our procedure for numerically calculating the absorption for complex materials is described in detail. Effects of off-diagonal terms in the Green's function and of the long-range ionic Coulomb interactions are examined. In particular, an absorption peak at $30 \mathrm{~cm}^{-1}$ is found to be especially sensitive to these contributions. This paper demonstrates (a) the theoretical existence of metastable phases of an alkali halide superlattice and (b) the application of anharmonic perturbation theory to determine the dielectric response in the infrared and millimeter-wave region for such complex materials. The particular structure studied is just one of many possibilities in this class of materials, which offers the opportunity for joint theoretical and experimental research to fabricate structures with properties tailored for specific applications.
\end{abstract}

\section{INTRODUCTION}

The infrared properties of alkali halides have been of interest as a testing ground for anharmonic theory and for technological applications, e.g., laser windows. Materials and/or devices with high sensitivity to electromagnetic radiation are important for technology. For infrared and higher frequencies specific applications can be tailored from materials with intrinsic excitations at the desired frequencies. Applications at microwave and lower frequencies become a matter of tailoring structures on a macroscopic scale. The region of transition between microwave and infrared is clearly more difficult. The purpose of these studies is (a) to explore, theoretically, the possibility of forming a superlattice of alkali halides, in particular, $\mathrm{RbF}(\mathrm{NaCl})_{2}$, and (b) to rigorously calculate the associated dielectric properties given such a superlattice. Superlattices of the sort reported here offer the possibility of tailoring at the microscopic level over selected distances, making such structures potentially useful for applications in this transition region. It would also be worthwhile to attempt to fabricate such materials for their use as a new testing ground for interionic potentials and perhaps to shed light on the supermodulus effect seen in intermetallic superlattices and not thoroughly understood. ${ }^{1}$ Other somewhat related exploratory types of calculations, but for simpler structures, have been performed by Hardy and co-workers. ${ }^{2,3}$

Both (a) and (b) depend crucially on the interionic potential. For this we use the Gordon-Kim (GK) theory which is an $a b$ initio theory for the interionic potential in closed-shell systems. ${ }^{4}$ It has been modified recently ${ }^{5}$ to allow for possible changes in ionic electron densities from those of free ions due to the presence of surrounding ions, but we base our work on the earlier theory in which one overlapped free-ion electron densities to obtain the electron density for any configuration of ions, and used the local-density approximation (LDA) including the Thomas-Fermi approximation for the kinetic energy term in the energy functional to obtain overlap interionic potentials. Within the GK theory, to a good approximation the interionic interactions are represented by two-bodycentral potentials, described by overlap and long-range interionic Coulomb terms. Numerical results for the overlap term are, of course, parametrized for the purpose of obtaining radial derivatives, but that parametrization can be done quite accurately.

The major computational effort is in the application of anharmonic perturbation theory (APT). APT lattice dynamics has been known for a long time, but its implementation has generally been restricted to rather simple systems. In most applications on simple systems, like alkali halides, only nearest-neighbor central forces are assumed for the explicit anharmonic potential derivatives, despite the fact that ionic Coulomb interactions are present. Plausibility arguments for the neglect of Coulomb in-

Work of the U. S. Government Not subject to U.S. copyright 
teractions in anharmonic terms had been given in the literature and perhaps their importance in anharmonic terms for alkali halides is still not widely appreciated, although their importance was discussed in a recent review article. $^{6}$ Indeed, we are aware of only three articles where Coulomb-interaction anharmonic contributions in ionic materials appear to have been rigorously taken into account. $^{7-9}$ The crystal structures treated are also generally of such simplicity that off-diagonal elements of the Green's function are ingorable if not zero and that the standard self-energy diagrams suffice. We mention that Maradudin and Fein ${ }^{10}$ presented an argument for neglecting off-diagonal self-energy terms within perturbation theory. On the other hand, it has been pointed out to us $^{11}$ that off-diagonal terms cannot be neglected if differences in the corresponding harmonic frequencies are small, i.e., roughly of the order of the off-diagonal selfenergy terms. Furthermore, we shall see that if there is a disparity among values of normal-mode electric polarization values then off-diagonal Green's-function elements are important even though they themselves may be small. This latter observation has also been made previously ${ }^{12}$ in the context of phenomenological treatments. Only Cowley ${ }^{13}$ has treated a system $\left(\mathrm{SrTiO}_{3}\right)$ in which off-diagonal elements in the phonon self-energy have been numerically evaluated and in which their effect on infrared properties has been displayed. Cowley's work was also unusual in the sense that the range of neighbors considered for evaluating anharmonic coefficients was not restricted to nearest neighbors. For structures in which every atom is a center of inversion the cubic anharmonic instantaneous-phonon self-energy term ${ }^{10}$ has been found to vanish; also, perhaps suggestive of a more general theorem, Haro et al. ${ }^{14}$ found that it vanishes in the case of a simple potential model for $\mathrm{Si}$, for which there are inversion sites, although not at the atomic positions. On the other hand, when the point group does not contain an inversion center this term clearly needs to be evaluated.

Maradudin and Ipatova ${ }^{15}$ and Birman ${ }^{16}$ have discussed the application of group representation theory to the phonon Green's function and, in particular, have shown that off-diagonal elements vanish unless the two polarization vectors involved correspond to the same partner of the same irreducible representation of the crystal point group in the case of zone-center modes. Earlier, Wallis et al. ${ }^{17}$ had also examined symmetry effects in Green's functions for zone-center optical modes, but they restricted their discussion to the rocksalt structure and did not make use of group representations. Wallis et al. proved that (a) no off-diagonal elements occur, (b) due to LO-TO splitting the LO- and TO-associated $\Gamma(\Omega)$ functions differ from one another, but only by a multiplicative constant, which is dependent on the ratio of LO and TO frequencies, and (c) the TO-mode degeneracy is not affected by anharmonicity, i.e., the $\Pi(\Omega)$ functions for both TO modes are the same. [We denote the complex self-energy function of frequency $(\Omega / 2 \pi)$ as $\Pi(\Omega)=\Delta(\Omega)-i \Gamma(\Omega)$.]

Formal aspects which we shall discuss, largely in Appendixes, are (a) the derivation of the expression for the phonon self-energy matrix corresponding to zone-center modes in terms of an average over an irreducible volume element of the first Brillouin zone (IBZ) and (b) the nature of the cubic anharmonic instantaneous-phonon selfenergy expression. In the appendixes and the following section, which gives the formulas to be evaluated, we will make brief use of the fact that the system of interest to us is trigonal, with point group $C_{3 v}$, and that its point and site-symmetry groups are identical. Section III contains a further development of the formulas and procedures of evaluation when two-body forces are assumed. (See Sec. IV mainly for further details of the structure which we have obtained.) Sections V and VI contain detailed checks on our numerical procedures and results for the phonon self-energies and dielectric properties. Section VII contains a summary.

\section{REVIEW OF THEORY}

Infrared properties are usually describable by the frequency-dependent complex dielectric susceptibility tensor $\chi_{\alpha \beta}(\Omega)$. Following the theoretical development of Cowley, ${ }^{18}$ Maradudin and Wallis, ${ }^{19}$ and Vinogradov ${ }^{20}$ we write, in the rigid-ion approximation,

$\chi_{\alpha \beta}(\Omega)=\frac{1}{k T V} \sum_{j, j^{\prime}=1}^{3 n} M_{\alpha}(0, j) M_{\beta}\left(0, j^{\prime}\right) G\left(0, j, j^{\prime} ; \Omega, T\right)$,

where

$$
M_{\alpha}(0, j)=\left(\frac{\hbar}{2 \omega(0, j)}\right)^{1 / 2} \sum_{\kappa=1}^{n} \frac{z_{\kappa}}{\left(M_{\kappa}\right)^{1 / 2}} e_{\alpha}(\kappa ; 0 j),
$$

and where $\omega(\mathbf{q}, j)$ and $\mathbf{e}(\kappa ; \mathbf{q}, j)(\kappa=1,2, \ldots, n)$ are the normal-mode frequency and polarization vector for a general wave vector $\mathrm{q}, z_{\kappa}$ and $M_{\kappa}$ are the ionic charge and mass of the $\kappa$ th basis ion, and $n$ is the number of basis ions. $G$ is the one-phonon frequency-dependent Green's function given by the Dyson equation,

$$
\begin{array}{r}
\frac{\hbar}{k T} \sum_{j^{\prime}}\left\{\left[\omega^{2}(\mathbf{q}, j)-\Omega^{2}\right] \delta_{j j^{\prime}}+4 \pi \omega(\mathbf{q}, j) \Pi\left(\mathbf{q}, j, j^{\prime} ; \Omega\right)\right\} \\
\times G\left(\mathbf{q}, j^{\prime}, j^{\prime \prime} ; \Omega\right)=2 \delta_{j j^{\prime \prime}} \omega(\mathbf{q}, j),
\end{array}
$$

and $\Pi\left(\mathbf{q}, j, j^{\prime} ; \Omega\right)$ is the self-energy function (in frequency units). We restrict ourselves to the quantity $\Pi_{j j^{\prime}}=\Pi\left(0, j, j^{\prime} ; \Omega\right)$. We will assume that in Eq. (1) $\mathbf{q}$ has approached zero along the trigonal $(z)$ direction and we will consider $\chi_{x x}$ only, which gives the dielectric response to radiation incident along the trigonal axis. The main difficulty of these calculations is the evaluation of the phonon self-energy diagram of Fig. 1(a) and we shall discuss its evaluation in detail. The other diagrams of importance are given in Figs. 1(b) and 1(c) but of these only the former, which is the instantaneous-phonon diagram, will be discussed in any detail. 


\section{A. Standard cubic anharmonic contribution}

Corresponding to the diagram of Fig. 1(a) we have the expression

$$
\begin{aligned}
\Pi_{j j^{\prime}}^{(3 a)} & =\Delta_{j j^{\prime}}^{(3 a)}-i \Gamma_{j j^{\prime}}^{(3 a)} \\
& =\sum_{\mathbf{q}_{1}, j_{1}, j_{2}} F^{(3 a)}(\Omega, \omega) \Phi^{*}\left(0, j ; \mathbf{q}_{1}, j_{1} ;-\mathbf{q}_{1}, j_{2}\right) \Phi\left(0, j^{\prime} ; \mathbf{q}_{1}, j_{1} ;-\mathbf{q}_{1}, j_{2}\right),
\end{aligned}
$$

where we write, symbolically,

$$
\begin{aligned}
F^{(3 a)}(\Omega, \omega)=- & \frac{1}{32 \pi N} \frac{\hbar}{\left[\omega(\mathbf{0}, j) \omega\left(\mathbf{0}, j^{\prime}\right)\right]^{1 / 2}} \frac{1}{\omega\left(\mathbf{q}_{1}, j_{1}\right) \omega\left(\mathbf{q}_{1}, j_{2}\right)} \\
\times & {\left[\int \pm \frac{n\left(\mathbf{q}_{1}, j_{1}\right)+n\left(\mathbf{q}_{1}, j_{2}\right)+1}{\left[\Omega \pm \omega\left(\mathbf{q}_{1}, j_{1}\right) \pm \omega\left(\mathbf{q}_{1}, j_{2}\right)\right]_{P}} \pm \frac{n\left(\mathbf{q}_{1}, j_{2}\right)-n\left(\mathbf{q}_{1}, j_{1}\right)}{\left[\Omega \pm \omega\left(\mathbf{q}_{1}, j_{1}\right) \mp \omega\left(\mathbf{q}_{1}, j_{2}\right)\right]_{P}}\right] } \\
& +i \pi\left\{ \pm\left[n\left(\mathbf{q}_{1}, j_{1}\right)+n\left(\mathbf{q}_{1}, j_{2}\right)+1\right] \delta\left(\Omega \mp \omega\left(\mathbf{q}_{1}, j_{1}\right) \mp \omega\left(\mathbf{q}_{1}, j_{2}\right)\right)\right. \\
& \left.\left. \pm\left[n\left(\mathbf{q}_{1}, j_{2}\right)-n\left(\mathbf{q}_{1}, j_{1}\right)\right] \delta\left(\Omega \mp \omega\left(\mathbf{q}_{1}, j_{1}\right) \pm \omega\left(\mathbf{q}_{1}, j_{2}\right)\right)\right\}\right],
\end{aligned}
$$

where $n \equiv\left(e^{\hbar \omega / k T}-1\right)^{-1}$ and $N$ is the number of unit cells. A major consideration of this calculation is the treatment of the cubic anharmonic coefficients,

$$
\begin{gathered}
\Phi\left(0, j ; \mathbf{q}_{1}, j_{1} ;-\mathbf{q}_{1}, j_{2}\right)=\sum_{\kappa} \sum_{l^{\prime}, \kappa^{\prime}} \sum_{l^{\prime \prime}, \kappa^{\prime \prime}} \frac{1}{\left(M_{\kappa} M_{\kappa^{\prime}} M_{\kappa^{\prime \prime}}\right)^{1 / 2}} \Phi_{\alpha \beta \gamma}\left[\begin{array}{lll}
0 & l^{\prime} & l^{\prime \prime} \\
\kappa & \kappa^{\prime} & \kappa^{\prime \prime}
\end{array}\right] e_{\alpha}(\kappa ; 0, j) e_{\beta}\left(\kappa^{\prime} ; \mathbf{q}_{1}, j_{1}\right) \\
\quad \times e_{\gamma}\left(\kappa^{\prime \prime} ;-\mathbf{q}_{1}, j_{2}\right) e^{i \mathbf{q}_{1} \cdot\left(\mathbf{x}_{l^{\prime} \kappa^{\prime}}-\mathbf{x}_{\left.l^{\prime \prime} \kappa^{\prime \prime}\right)}\right)}
\end{gathered}
$$

where

$$
\Phi_{\alpha \beta \gamma}\left(\begin{array}{lll}
0 & l^{\prime} & l^{\prime \prime} \\
\kappa & \kappa^{\prime} & \kappa^{\prime \prime}
\end{array}\right)
$$

is a cubic anharmonic coupling coefficient. (The Einstein summation convention over repeated indices is used for

(a)

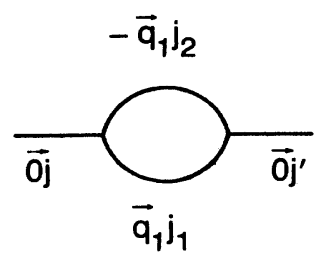

(c)

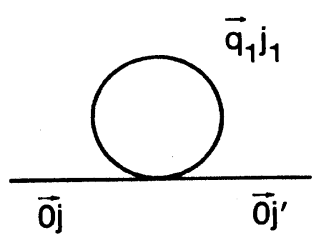

FIG. 1. Lowest-order anharmonic self-energy diagrams. (a) Standard cubic anharmonic diagram; (b) instantaneous-phonon cubic anharmonic diagram; (c) standard quartic anharmonic diagram.

(b)

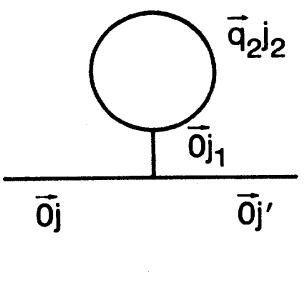

$$
\times \Phi\left(0, j_{1} ; \mathbf{q}_{2}, j_{2} ;-\mathbf{q}_{2}, j_{2}\right),
$$

where

$$
\begin{gathered}
F^{(3 b)}(\omega)=- \\
\frac{1}{16 \pi N} \frac{1}{\left[\omega(0, j) \omega\left(0, j^{\prime}\right)\right]^{1 / 2}} \\
\times \frac{2 n\left(q_{2}, j_{2}\right)+1}{\omega\left(0, j_{1}\right)^{2} \omega\left(\mathbf{q}_{2}, j_{2}\right)} .
\end{gathered}
$$

As is known (e.g., Ref. 6), both with respect to its realness and independence of $\Omega, \Pi^{(3 b)}$ is similar to the ordinary quartic anharmonic contribution and dissimilar to the ordinary cubic anharmonic contribution.

We observe that the $q=0$ internal phonon line in Fig. 1 (b) appears to render the results dependent on the direction of $q$ used in taking that $q=0$ limit, since the Coulomb term in the dynamical matrix is irregular at small $q$. In fact, for any finite crystal, momentum conservation at each interaction vertex is not required and an average [of Eq. (8)] over wave-vector directions, dependent on sample shape, is appropriate. On the other hand, when retardation effects of the electromagnetic interaction between ions are taken into account there exist 
unambiguous $q=0$ frequencies and polarization vectors, i.e., ones which do not depend on the limiting process, as proven in the case of a diatomic system by Loudon. ${ }^{21}$ These modes have polarization either parallel or perpendicular to the trigonal axis, and they have LO-mode frequencies. In Appendix B we use group theory to prove that only the modes which have polarization parallel to the trigonal axis contribute to Eq. (8). It is also demonstrated in Appendix B that in Eq. (8) the summation over the $\mathrm{BZ}$ can simply be replaced by a summation over the IBZ.

\section{PAIR-POTENTIAL SIMPLIFICATIONS AND DETAILS OF PROCEDURES}

Next we assume that the potential energy, $\Phi$, can be written as the sum of pair potentials. We may write, for example, $\Phi_{\alpha \beta \gamma}^{p}(1,1,2)$ as the contribution to $\Phi_{\alpha \beta \gamma}(1,1,2)$ from the pair potential, $\phi(r)$, between atoms at sites 1 and 2. Following Leibfried and Ludwig ${ }^{22}$ we obtain

$$
\begin{aligned}
\Phi_{\alpha \beta \gamma}^{p}(1,1,2)= & \left(\delta_{\alpha \gamma} X_{\beta}^{21}+\delta_{\beta \gamma} X_{\alpha}^{21}+\delta_{\alpha \beta} X_{\gamma}^{21}\right) \mathcal{O}^{2} \phi \\
& +X_{\alpha}^{21} X_{\beta}^{21} X_{\gamma}^{21} \mathcal{O}^{3} \phi
\end{aligned}
$$

where $\mathbf{X}^{21}=\mathbf{X}(2)-\mathbf{X}(1)$ and

$$
\mathcal{O}^{n} \equiv\left(\frac{1}{r} \frac{\partial}{\partial r}\right)^{n}
$$

Other anharmonic coefficients derived from $\phi(r)$ that enter the sums in Eq. (7) are $\Phi_{\alpha \beta \gamma}^{p}(1,1,1), \Phi_{\alpha \beta \gamma}^{p}(1,2,1)$, and $\Phi_{\alpha \beta \gamma}^{p}(1,2,2)$ assuming atom 1 is in the cell $l=0$ and atom 2 is not. Otherwise, if both atoms are in cell $l=0$, terms of the form $\Phi_{\alpha \beta \gamma}^{p}(2,1,1)$, etc., contribute to the sum as well. However, it is necessary to evaluate expression (10) only once for each ion pair since we can make use of the simple relations: ${ }^{22}$

$$
\begin{aligned}
& \Phi_{\alpha \beta \gamma}^{p}(1,1,1)=-\Phi_{\alpha \beta \gamma}^{p}(1,1,2), \\
& \Phi_{\alpha \beta \gamma}^{p}(1,2,1)=\Phi_{\alpha \beta \gamma}^{p}(1,1,2), \\
& \Phi_{\alpha \beta \gamma}^{p}(1,2,2)=-\Phi_{\alpha \beta \gamma}^{p}(1,1,2), \\
& \Phi_{\alpha \beta \gamma}^{p}(2,1,1)=-\Phi_{\alpha \beta \gamma}^{p}(1,1,2),
\end{aligned}
$$

etc., derived on the assumption of a central potential. Rather than obtaining algebraic expressions that are applicable to a specified structure and range of interactions, our general computational procedure is to search for all pairs of atoms of separation less than some maximum distance, with one atom of each pair restricted to $l=0$, and to numerically evaluate the various contributions to the sum in Eq. (7) for each pair. Furthermore, it may be advisable to make use of Fourier transforms of

$$
\Phi_{\alpha \beta \gamma}\left[\begin{array}{ccc}
0 & l^{\prime} & l^{\prime \prime} \\
\kappa & \kappa^{\prime} & \kappa^{\prime \prime}
\end{array}\right]
$$

Similar transforms, but written expressly for central forces, were defined in Refs. 6 and 7.

The anharmonic coupling coefficients appearing in expression (5) can be written as

$$
\Phi\left(0, j ; \mathbf{q}_{1}, j_{1} ;-\mathbf{q}_{1}, j_{2}\right)=\sum_{\kappa, \kappa^{\prime}, \kappa^{\prime \prime}} \frac{1}{\left(M_{\kappa} M_{\kappa^{\prime}} M_{\kappa^{\prime \prime}}\right)^{1 / 2}} e_{\alpha}(\kappa ; 0, j) e_{\beta}\left(\kappa^{\prime} ; \mathbf{q}_{1}, j_{1}\right) e_{\gamma}\left(\kappa^{\prime \prime} ;-\mathbf{q}_{1}, j_{2}\right) \Phi_{\alpha \beta \gamma}\left(\mathbf{q}_{1}\right)
$$

where

$$
\begin{aligned}
\boldsymbol{\Phi}_{\substack{\alpha \beta \gamma \\
\kappa \kappa^{\prime} \kappa^{\prime \prime}}}\left(\mathbf{q}_{1}\right) & \equiv \sum_{l^{\prime}, l^{\prime \prime}} \Phi_{\alpha \beta \gamma}\left[\begin{array}{lll}
0 & l^{\prime} & l^{\prime \prime} \\
\kappa & \kappa^{\prime} & \kappa^{\prime \prime}
\end{array}\right] e^{i \mathbf{q}_{1} \cdot\left(\mathbf{X}_{l^{\prime} \kappa^{\prime}}-\mathbf{X}_{l^{\prime \prime} \kappa^{\prime \prime}}\right)} \\
& =\sum_{p} \sum_{l^{\prime}, l^{\prime \prime}} \Phi_{\alpha \beta \gamma}^{p}\left[\begin{array}{lll}
0 & l^{\prime} & l^{\prime \prime} \\
\kappa & \kappa^{\prime} & \kappa^{\prime \prime}
\end{array}\right] e^{i \mathbf{q}_{1} \cdot\left(\mathbf{X}_{l^{\prime} \kappa^{\prime}}-\mathbf{X}_{l^{\prime \prime} \kappa^{\prime \prime}}\right)} .
\end{aligned}
$$

The sum over $p$ goes over ion pairs. The $l^{\prime}, l^{\prime \prime}$ summations in the latter expression reduce to at most a single nonvanishing term for each $p$. For example the pair $p=1$, say, corresponding to sites $\left(\begin{array}{l}0 \\ \kappa\end{array}\right)$ and $\left(\begin{array}{l}l \\ \kappa^{\prime}\end{array}\right)$ gives a nonvanishing contribution to $\Phi_{\alpha \beta \gamma}\left(\mathbf{q}_{1}\right)$ of

$$
\Phi_{\alpha \beta \gamma}^{(1)}\left(\begin{array}{ccc}
0 & l & 0 \\
\kappa & \kappa^{\prime} & \kappa
\end{array}\right) e^{i \mathbf{q}_{1} \cdot\left(\mathbf{X}_{l \kappa^{\prime}}-\mathbf{X}_{\kappa}\right)} .
$$

The use of such Fourier transforms is economical because the $p$ summation can be performed independently of the $j_{1}, j_{2}$ summations in Eq. (5). Also, it is known that if $\phi(r)$ is chosen as the ionic Coulomb potential, the summations defining these Fourier transforms are absolutely convergent, unlike the harmonic-approximation case. Hence, a real-space sum is sufficient, although it is possible to make use of theta transformations as done in previous work. ${ }^{8}$

Furthermore, if the range of the interatomic potential is not large compared to the size of the unit cell it may not be beneficial to utilize Fourier transformations. In that case it is useful to write expression (5) as

$$
\Pi_{j j^{\prime}}=\sum_{q_{1}, j_{1}, j_{2}} \sum_{\kappa, \kappa^{\prime}} F^{(3 a)}(\Omega, \omega) \widetilde{e}_{\alpha}(\kappa ; \mathbf{0}, j) \widetilde{e}_{\alpha^{\prime}}\left(\kappa^{\prime} ; \mathbf{0}, j^{\prime}\right) G_{\alpha}\left(\kappa ; \mathbf{q}_{1}, j_{1} ;-\mathbf{q}_{1}, j_{2}\right) G_{\alpha^{\prime}}^{*}\left(\kappa^{\prime} ; \mathbf{q}_{1}, j_{1} ;-\mathbf{q}_{1}, j_{2}\right),
$$

where 


$$
\begin{aligned}
& G_{\alpha}\left(\kappa ; \mathbf{q}_{1}, j_{1} ;-\mathbf{q}_{1}, j_{2}\right) \\
& \equiv \sum_{p} \sum_{l^{\prime}, \kappa^{\prime}} \sum_{l^{\prime \prime}, \kappa^{\prime \prime}} \Phi_{\alpha \beta \gamma}^{p}\left[\begin{array}{ccc}
0 & l^{\prime} & l^{\prime \prime} \\
\kappa & \kappa^{\prime} & \kappa^{\prime \prime}
\end{array}\right] \widetilde{e}_{\beta}\left(\kappa^{\prime} ; \mathbf{q}_{1}, j_{1}\right) \widetilde{e}_{\gamma}\left(\kappa^{\prime \prime} ;-\mathbf{q}_{1}, j_{2}\right) e^{i \mathbf{q}_{1} \cdot\left(\mathbf{x}_{l^{\prime}}-\mathbf{x}_{l^{\prime \prime}}\right)} \\
& =\sum_{p}^{\prime} \Phi_{\alpha \beta \gamma}^{p}\left[\begin{array}{ccc}
0 & 0 & l^{(p)} \\
\kappa & \kappa & \kappa_{p}
\end{array}\right]\left[\begin{array}{l}
{\left[\widetilde{e}_{\beta}\left(\kappa ; \mathbf{q}_{1}, j_{1}\right) \widetilde{e}_{\gamma}\left(\kappa_{p} ;-\mathbf{q}_{1}, j_{2}\right) e^{-i \mathbf{q}_{1} \cdot \mathbf{x}_{l}(p)}+\widetilde{e}_{\beta}\left(\kappa_{p} ; \mathbf{q}_{1}, j_{1}\right) \widetilde{e}_{\gamma}\left(\kappa ;-\mathbf{q}_{1}, j_{2}\right) e^{i \mathbf{q}_{1} \cdot \mathbf{x}_{l}(p)}\right.} \\
\left.-\widetilde{e}_{\beta}\left(\kappa ; \mathbf{q}_{1}, j_{1}\right) \widetilde{e}_{\gamma}\left(\kappa ;-\mathbf{q}_{1}, j_{2}\right)-\widetilde{e}_{\beta}\left(\kappa_{p} ; \mathbf{q}_{1}, j_{1}\right) \widetilde{e}_{\gamma}\left(\kappa_{p} ;-\mathbf{q}_{1}, j_{2}\right)\right]
\end{array}\right.
\end{aligned}
$$

and where the prime on the summation sign denotes that the sum is restricted to ion pairs for which one of the sites is $\left(\begin{array}{c}0 \\ \kappa\end{array}\right)$; the others are denoted $\left(l_{\kappa_{p}}^{(p)}\right)$. In the above expressions we have also used the definition

$$
\widetilde{\mathbf{e}}(\kappa ; \mathbf{q}, j) \equiv \frac{e^{i \mathbf{q} \cdot \mathbf{X}_{\kappa}}}{\left(M_{\kappa}\right)^{1 / 2}} \mathbf{e}(\kappa ; \mathbf{q}, j)
$$

and expressions (11). To reduce computational time, use can be made of the fact that the $j_{1}, j_{2}$ sums in Eq. (5) can be replaced by sums for which $j_{1} \geq j_{2}$, as can easily be shown. This replacement depends on the fact that in the $q_{1}$ sum $q_{1}$ runs over $-q$ as well as $q$; one can still make use of it when the sum is restricted to the IBZ (as in Appendix $\mathrm{A}$ ), and one must still consider only the real part of the product following the $F^{(3 a)}$ factor.

Finally, we note that similar arguments to those presented for cubic anharmonicity also hold true for the quartic case, which will therefore not be discussed in any detail. Suffice it to say that the quartic case is an easier computational problem, because it involves single, rather than double, summations over polarization indices [see Fig. 1(c)] and is $\Omega$ independent.

\section{MATERIAL STRUCTURAL CONSTANTS AND PHONON DISPERSION CURVES}

The calculations of infrared properties to be presented are based on a structure of a material which to our knowledge does not presently exist in nature but one which we have found to be metastable on the basis of the GK theory. In particular the theoretical compound which we study is $\mathrm{RbF}(\mathrm{NaCl})_{2}$. This material was chosen because the GK theory has been found to yield reasonably good thermal and structural equilibrium properties of $\mathrm{NaCl}$ and $\mathrm{RbF}$, and because the lattice parameters of $\mathrm{NaCl}$ and $\mathrm{RbF}$ are similar which we presumed to be a favorable circumstance for stability of the combined compound. The two-to-one chemical ratio is a rather arbitrary choice, however. The structure which we anticipated was basically the rocksalt structure, i.e., planes of identical ions stacked along the 111 axis, identically to rocksalt, in the sequence $\mathrm{Na}^{+}-\mathrm{Cl}^{-}-\mathrm{Na}^{+}-\mathrm{Cl}^{-}-\mathrm{Rb}^{+}-$ $\mathrm{F}^{-}-\mathrm{Na}^{+}$, etc. However, this (trigonal) structure was found to be dynamically unstable. On the other hand, a metastable trigonal structure of point group, $C_{3 v}$, with the same ionic stacking sequence as above was obtained through a fortunate choice of stacking-perhaps one of several possible ones. This latter structure is distinguished from the rocksalt stacking by a displacement of the $\mathrm{F}^{-}$ions from their "rocksalt" positions to the same in-plane sites as those of the adjacent $\mathrm{Na}^{+}$layer. We found this stacking to lead to an anomalously lowlying transverse acoustic-phonon branch along the trigonal direction of propagation. The structural parameters were found through a minimization of $\Phi$, with respect to the interplanar distances and basal plane lattice parameter, i.e., the above stacking sequence and intraplanar arrangement of ions was assumed fixed. The results of this evaluation are the following: The hexagonal unit-cell lattice parameters are $a=4.041 \AA$ and $c=10.223 \AA$; the $c$ axis components of the basis-ion positions are $0,2.237$, $3.610,5.428,6.813$, and $8.856 \AA$ corresponding to $\mathrm{Rb}^{+}$, $\mathrm{Cl}^{-}, \mathrm{Na}^{+}, \mathrm{Cl}^{-}, \mathrm{Na}^{+}$, and $\mathrm{F}^{+}$, respectively. Through a molecular-dynamics (MD) calculation, this structure was also found to be stable with respect to a temperature of at least $100 \mathrm{~K}$, and indeed an MD pressure ${ }^{23}$ was necessary to cause the structure to transform to a more stable $[\Delta \Phi=0.04 \mathrm{eV} /$ (ion pair)] triclinic one. We also note that the structure upon which we shall base our calculations, i.e., the above dynamically stable trigonal one, has a higher energy by $0.07 \mathrm{eV} /$ ion-pair than the abovementioned "rocksalt" structure (with ion positions for which the energy was a minimum). Since this "rocksalt" structure is dynamically unstable there must exist an even lower energy structure for our compound than those considered here. Therefore, it appears that although the structure of our choice is indeed metastable it might require a great deal of ingenuity to synthesize it. On the other hand, our results suggest that a rich assortment of phases are possible within this system, especially if the relative amounts of $\mathrm{NaCl}$ and $\mathrm{RbF}$ were varied.

Phonon dispersion curves are displayed in Fig. 2. The low-lying $\Delta$ direction transverse acoustic branch represents rigid-layer motion and is characteristic of the unusual structural property described in the previous paragraph. The discontinuities at the $\Gamma$ point of the Brillouin zone are "LO-TO" splitting effects and are known to occur in ionic materials with complex structures. Arrows in the figure show two of those splittings. Away from the $\Gamma$ point, in the $\Delta$ direction, only the dotted lines represent purely transverse modes as the other lines represent partially transverse and longitudinal modes. It is also evident that substantially lower TO frequencies occur for the compound than for individual alkali halides. For a consistent comparison with the results in Fig. 2 we have used the GK theory to obtain the values, $163,211,132$, and $181 \mathrm{~cm}^{-1}$ for $\mathrm{NaCl}, \mathrm{NaF}, \mathrm{RbCl}$, and $\mathrm{RbF}$, respectively. These are determined at the theoretical room-temperature equilibrium volumes ${ }^{24}$ and are seen 


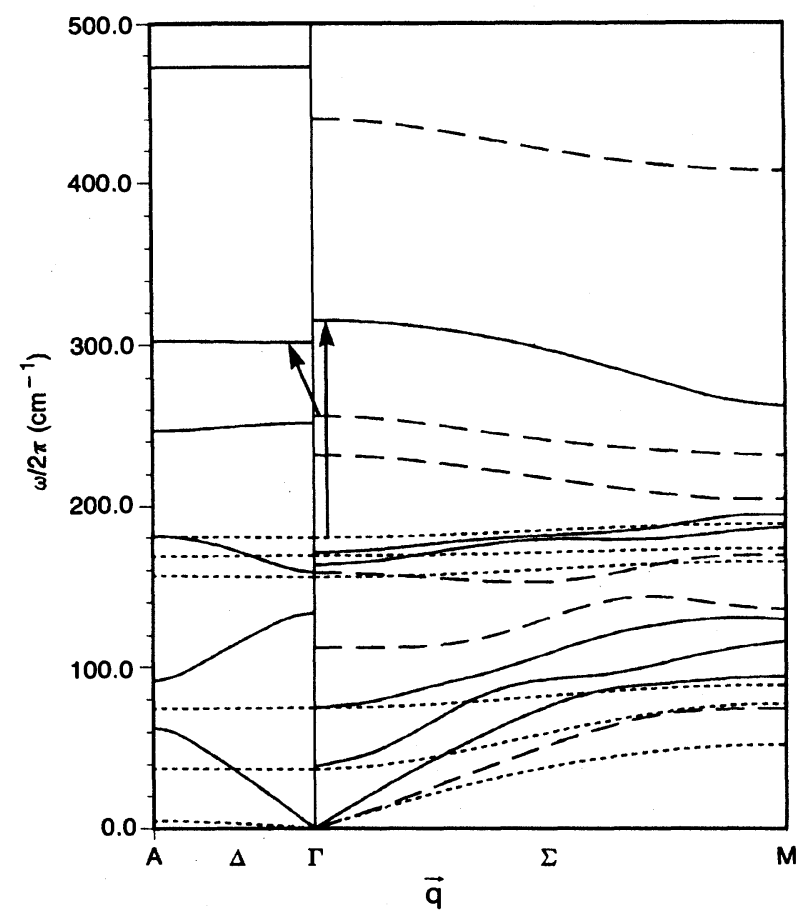

FIG. 2. Dispersion curves along $\Sigma$ and $\Delta$. Modes along $\Delta$ are purely longitudinal (solid) and transverse (dotted). Modes along $\Sigma$ are both purely basal-plane polarized transverse (dotted) and mixed: solid (dashed) lines correspond to pure longitudinal (transverse) modes only in the vicinity of the $\Gamma$ point.

to be substantially above the mixed compound's lowest TO frequency. The former values can also be seen to be in reasonable agreement with the respective experimen$\mathrm{tal}^{25}$ values of $164,239,116$, and $156 \mathrm{~cm}^{-1}$. LO-TO splittings similarly obtained by theory are 160, 262, 89, and $145 \mathrm{~cm}^{-1}$ to be compared with the experimental ${ }^{25}$ values of $100,175,57$, and $130 \mathrm{~cm}^{-1}$ for $\mathrm{NaCl}, \mathrm{NaF}, \mathrm{RbCl}$, and $\mathrm{RbF}$, respectively. The too large overall theoretical LOTO splittings are not surprising since we use a rigid-ion model. While a detailed calculation is necessary to obtain the infrared properties one can obtain a preliminary estimate for the relative strengths of the absorption by considering the quantity $\boldsymbol{M}_{\boldsymbol{x}}$ [defined in Eq. (2)] for the various TO zone-center modes. For TO modes in order of increasing frequency these are $-1.30,0.30,4.27$, -1.83 , and 5.02 in units of $10^{-17}$ esu cm. Given the fact that the two lowest-lying modes are essentially zonefolded modes it is not surprising that they have weaker absorption strengths than the remaining ones.

\section{CALCULATIONAL DETAILS AND RESULTS FOR $\Gamma$ AND $\Delta$}

\section{A. Preparation of the zone-center modes}

The polarization vectors were calculated for small $q$ with $q$ approximately parallel to the $c$ axis (we set $q_{z} / q=0.99$ for technical reasons). At $q=0$ it is valid and somewhat simplifying to choose the polarization vectors to be real, as we have done in the above expressions. However, because of an arbitrariness in the overall phase factor a particular diagonalization routine will not necessarily yield real $q=0$ polarization vectors. In our case it was necessary to multiply the polarization vectors resulting from the diagonalization by a different phase factor for each $3 n$-dimensional polarization vector. Small residual imaginary components are present in our results because of the above procedures. Additionally, we need to work with polarization vectors of different sets of degenerate modes that transform amongst each other identically under point-group operations. Again, this transformation property must be invoked subsequent to the diagonalization. In our case this was accomplished by rotations in the basal plane, a valid procedure because all of the degenerate modes have polarization in that plane. Finally, we made checks to ensure that our final results were reasonably independent of the particular small value of $q$ chosen for our "zone-center" modes. We estimate that these numerical approximations lead to negligible ( $\lesssim 1 \%$ ) errors in the results for $M_{x}$ and the self-energies.

\section{B. Discussion of grids in q space}

We have used the Brillouin-zone sampling method for performing all summations within the IBZ. Two uniform grids of points, defined as follows, were primarily used:

$$
\begin{aligned}
& q_{x}=n_{x} \pi a^{*} / N \\
& q_{y}=n_{y} \pi a^{*} / N+\pi a^{*} / 2 N, \\
& q_{z}=n_{z} \pi c^{*} / M+\pi c^{*} / 2 M,
\end{aligned}
$$

where $\mathbf{a}^{*}$ and $\mathbf{c}^{*}$ are (hexagonal) reciprocal-lattice vectors, $n_{i}$ are non-negative integers, and $N$ and $M$ were chosen to be 6 and 2 for one grid $(A)$ and 12 and 4 for the other $(B)$ thus yielding approximately cubic grids consisting of 47 and 357 points in the IBZ, respectively. Contributions from IBZ boundary points were weighted appropriately. The above grids contain no points on the $q_{z}$ axis, in order to exclude disproportionately large effects of the anomalously low $\Delta$ TA phonons. We have justified the use of these grids through a few calculations with the use of very fine $\left(q_{x}-q_{y}\right)$ grids in a small region of $q_{x}-q_{y}$ space in the vicinity of the $q_{z}$ axis. Specifically, the anomalous branch was found to yield negligible contributions due to the small amount of $q$ space over which the anomaly extends. Nevertheless, we also present brief results for a grid $(C)$ of the same fineness as grid $B$ but for which the term, $\pi a^{*} / 2 N$, in the expression for $q_{y}$ is absent. The difference in results between grid $C$ and either grids $A$ or $B$ (for which results are generally in excellent agreement) represents an overestimate of the uncertainty in the results since the effects of the anomalous phonon branch are exaggerated by the use of grid $C$.

\section{Discussion of effects of numerical approximations and results for $\underline{\Gamma}$ and $\underline{\Delta}$}

It is important to examine the sensitivity of the results for the real and imaginary parts, $\underline{\Delta}$ and $\underline{\Gamma}$, of the phonon 

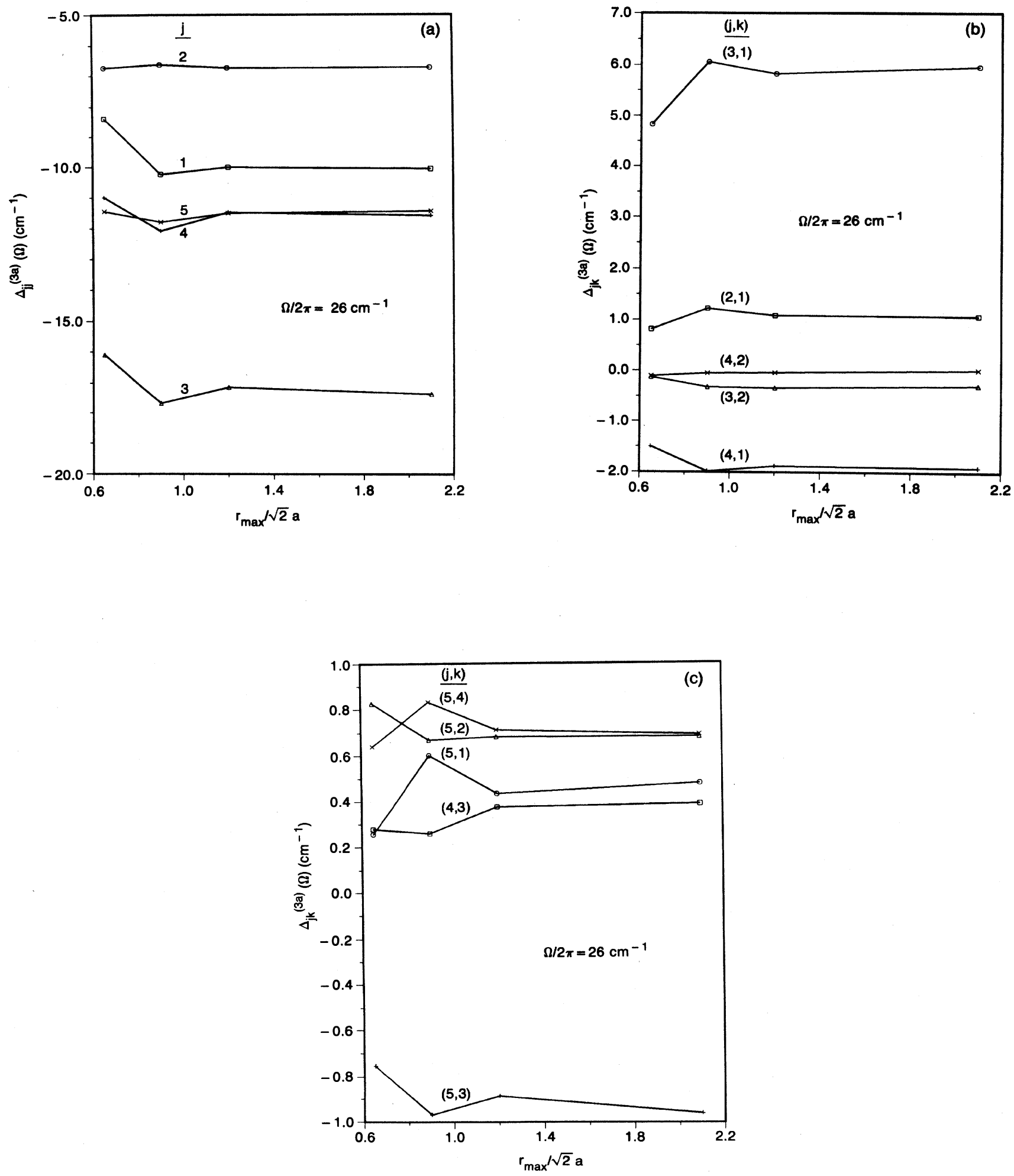

FIG. 3. Effect of cutoff in range of interionic potential on $\Delta_{j k}^{(3 a)}(\Omega)$ at $\Omega / 2 \pi=26 \mathrm{~cm}^{-1}$. Based on $\epsilon=3.33 \mathrm{~cm}^{-1}$. Diagonal elements are in (a) and off-diagonal elements in (b) and (c). Lines merely connect calculated points. Note differences of frequency scales among (a), (b), and (c). 
TABLE I. Diagonal components of $\Omega$-independent contributions to self-energy.

\begin{tabular}{ccccrrr}
\hline & \multicolumn{5}{c}{$r_{\max }$} & \multicolumn{5}{c}{$\Delta_{j j}^{(4)}\left(\Delta_{j j}^{3 b}\right)\left(\mathrm{cm}^{-1}\right)$} \\
Grid & $(\sqrt{2} a)$ & $j=1$ & $j=2$ & \multicolumn{1}{c}{$j=3$} & \multicolumn{1}{c}{$j=4$} & \multicolumn{1}{c}{$j=5$} \\
\hline$B$ & 0.65 & 10.95 & 6.28 & 10.29 & 6.73 & \multicolumn{1}{l}{9.03} \\
$A$ & 0.65 & $10.80(-4.54)$ & $6.28(-0.67)$ & $10.23(-2.48)$ & $6.72(-0.63)$ & $9.03(-0.62)$ \\
$A$ & 0.90 & $(-5.82)$ & $(-0.85)$ & $(-2.80)$ & $(-0.54)$ & $(-0.97)$ \\
$A$ & 1.20 & $11.09(-5.64)$ & $6.44(-0.82)$ & $10.33(-2.82)$ & $6.82(-0.56)$ & $9.07(-0.86)$ \\
$A$ & $0.65^{\mathrm{a}}$ & $23.02(-17.86)$ & $6.28(-2.59)$ & $15.10(-7.91)$ & $7.26(-1.20)$ & $9.01(-1.56)$ \\
\hline \hline
\end{tabular}

${ }^{a}$ Ionic Coulomb contributions were excluded from the anharmonic coefficients in obtaining the values in this row.

self-energy, to various numerical constants that determine the accuracy of the calculations. In addition to the grid-size parameters defined previously we need to consider the sensitivity to the value of $\epsilon$, which is used to represent ${ }^{26}$ both the Dirac delta function and principalvalue function entering $F^{(3 a)}(\Omega, \omega)$ [Eq. (5)], and to the value of $r_{\max }$, the maximum interionic distance for which anharmonic potential derivatives are evaluated. In addition, we shall also consider the effect on our results of the neglect of the interionic Coulomb interaction in the anharmonic coefficients. Selected results will be presented in order to illustrate the various dependencies and only $T=300 \mathrm{~K}$, TO mode self-energies are considered.

\section{Convergence and ionic Coulomb effects}

First we consider the effect of cutting off the interatomic potential at a finite interatomic distance, $r_{\max }$. Figure 3 shows the $r_{\max }$ dependencies of standard-cubicanharmonic (SCA) $\Delta$ at $\Omega / 2 \pi=26 \mathrm{~cm}^{-1}$ and $\epsilon=3.33$ $\mathrm{cm}^{-1}$. (The TO mode indices, $j$ and $k$, are ordered according to frequency.) It appears that convergence has been achieved to within a few percent. We note that the elements of most interest to us are those for which one of the indices corresponds to the lowest frequency mode since we are primarily interested in obtaining lowfrequency absorption properties. Cutoff effects in quartic and instantaneous-phonon cubic anharmonic contributions are given in Tables I and II. The effects of neglecting the Coulomb interaction in SCA $\Delta$ are given in Fig. 4. It should be mentioned that to within the accuracies of the tabulated values and the scales of the figures all $r_{\max }$ effects in these results arise from the ionic Coulomb interactions. It is seen from these results that the $r_{\max }$ effect is less in the quartic contributions than in the cubic ones, as expected since the quartic terms involve a higher radial derivative and hence are more "short-ranged" than the cubic terms. Also, the lowest frequency mode $(j=1)$ is seen to be the one most affected by both the value of $r_{\max }$ and the inclusion of the Coulomb interaction. Indeed, if the Coulomb potential is not included then the net $j=1$ frequency shift is sufficient to yield an instability in the sense that the imaginary part of the susceptibility function becomes peaked at $\Omega=0.0$. Obviously, there also is a corresponding large effect in $\Gamma_{11}(\Omega)$.

\section{Grid and Lorentzian-broadening effects}

It is known that there is a close connection between ( $q$ space) grid and $\epsilon$ dependencies. In particular, for a given

TABLE II. Off-diagonal components of $\Omega$-independent contributions to self-energy.

\begin{tabular}{ccccccc}
\hline \hline & \multicolumn{5}{c}{$\Delta_{j k}^{(4)}\left(\Delta_{j k}^{3 b}\right)\left(\mathrm{cm}^{-1}\right)$} \\
Grid & $(\sqrt{\max } a)$ & $j k=21$ & $j k=31$ & $j k=32$ & $j k=41$ & $j k=42$ \\
\hline$B$ & 0.65 & -1.76 & -5.93 & 0.63 & 1.73 & 0.81 \\
$A$ & 0.65 & $-1.74(0.75)$ & $-5.83(2.77)$ & $0.62(-0.47)$ & $1.70(-1.00)$ & $0.81(-0.0)$ \\
$A$ & 0.90 & $(0.99)$ & $(3.53)$ & $(-0.59)$ & $(-1.26)$ & $(0.01)$ \\
$A$ & 1.20 & $-1.88(0.95)$ & $-5.98(3.44)$ & $0.66(-0.54)$ & $1.71(-1.21)$ & $0.90(-0.01)$ \\
$A$ & $0.65^{\mathrm{a}}$ & $-3.60(2.67)$ & $-13.67(10.89)$ & $1.90(-1.69)$ & $4.63(-3.86)$ & $0.35(-0.04)$ \\
& & & & & \\
Grid & $r_{\max }$ & $(\sqrt{2} a)$ & $j k=43$ & $j k=51$ & $\Delta_{j k}^{(4)}\left(\Delta_{j k}^{3 b}\right)\left(\mathrm{cm}^{-1}\right)$ & \\
\hline$B$ & 0.65 & -1.09 & $0.49=52$ & $j k=53$ & $j k=54$ \\
$A$ & 0.65 & $-1.06(0.54)$ & $0.51(0.0)$ & $-1.95(0.0)$ & $0.66(0.0)$ & $-0.97(0.0)$ \\
$A$ & 0.90 & $(0.66)$ & $(0.0)$ & $(0.0)$ & $(0.0)$ & $(0.0)$ \\
$A$ & 1.20 & $-1.12(0.67)$ & $0.49(0.0)$ & $-1.94(0.0)$ & $0.65(0.0)$ & $-0.94(0.0)$ \\
$A$ & $0.65^{\mathrm{a}}$ & $-2.95(2.30)$ & $-0.94(-0.02)$ & $-1.66(0.02)$ & $1.55(-0.01)$ & $-1.22(-0.01)$ \\
\hline \hline
\end{tabular}

${ }^{\text {a }}$ See footnote in Table $\mathbf{I}$. 
TABLE III. Diagonal components of SCA contribution to $\Delta_{i j}\left(\omega_{j}\right)$. Based on results for grid $A$ and $\epsilon=3.33 \mathrm{~cm}^{-1}$.

\begin{tabular}{rrc}
\hline $\begin{array}{c}\omega_{j} / 2 \pi \\
\left(\mathrm{cm}^{-1}\right)\end{array}$ & $\begin{array}{c}\Delta_{j j}^{(3 a)}\left(\mathrm{cm}^{-1}\right) \\
36.9\end{array}-10.45$ & Total $\left[\Delta_{j j}\left(\omega_{j}\right) \equiv \Delta_{j j}^{(3 a)}\left(\omega_{j}\right)+\Delta_{j j}^{(3 b)}+\Delta_{j j}^{(4)}\right]$ \\
$\left(\mathrm{cm}^{-1}\right)$
\end{tabular}

grid, sensible results can be obtained only for a finite range of values of $\epsilon$. Ideally, for infinitely large crystals and a narrow frequency resolution, we desire results for the limits of an infinitely fine grid and $\epsilon=0$ with the limits taken in that order. Results in Figs. 5(a) and 5(b) show that, for our range of grid parameters and values of $\epsilon$ (a) merely larger amplitude oscillations are introduced into $\Gamma_{11}(\Omega)$ as one goes to smaller $\epsilon$ (for fixed grid parameters), and (b) larger-amplitude, longer-period, oscillations are introduced into $\Gamma_{11}(\Omega)$ as one goes to a cruder grid (for a fixed value of $\epsilon$ ). Grid effects in other quantities are shown in Tables I and II and Fig. 4. As previously discussed, we believe that the difference in results between grid $A$ and grid $C$ (shown in Fig. 4 ) is greater than uncertainties resulting from the use of finite grids because of the anomalously large contribution from the lowfrequency TA modes, contributing for grid $C$ and not for grid $A$. Instead we believe that the grid- $A$-to-grid- $B$ differences shown in Fig. 5(b) and Tables I and II are representative of our uncertainties.

\section{SCA results}

Complete results for SCA contributions to $\Delta$ and $\Gamma$ are presented for particular values of $\epsilon\left(3.33 \mathrm{~cm}^{-1}\right)$ and $r_{\max }$

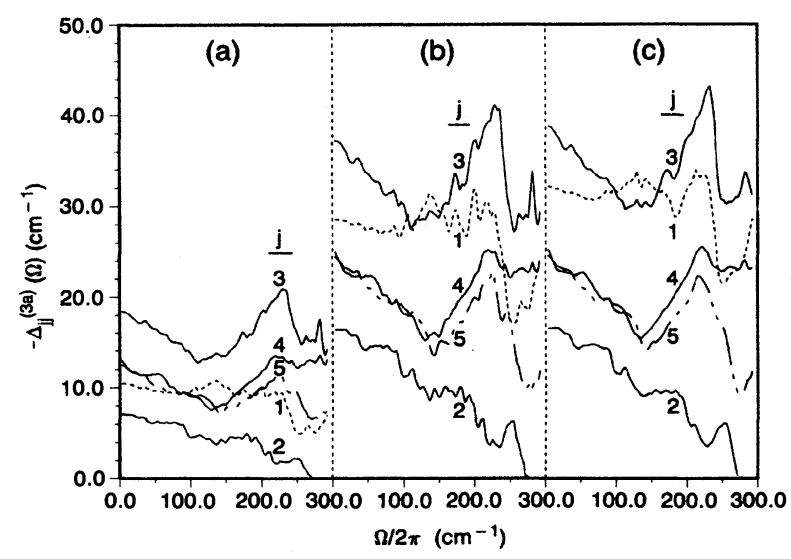

FIG. 4. Effects in $\Delta_{j j}^{(3 a)}(\Omega)$ of ionic Coulomb interaction and of use of q-space grid $C$. (a) Grid $A$ results; (b) grid $A$ results for which explicit ionic Coulomb contributions to the anharmonic potential energy coefficients were excluded; (c) same as (b) except that grid $C$ was employed.
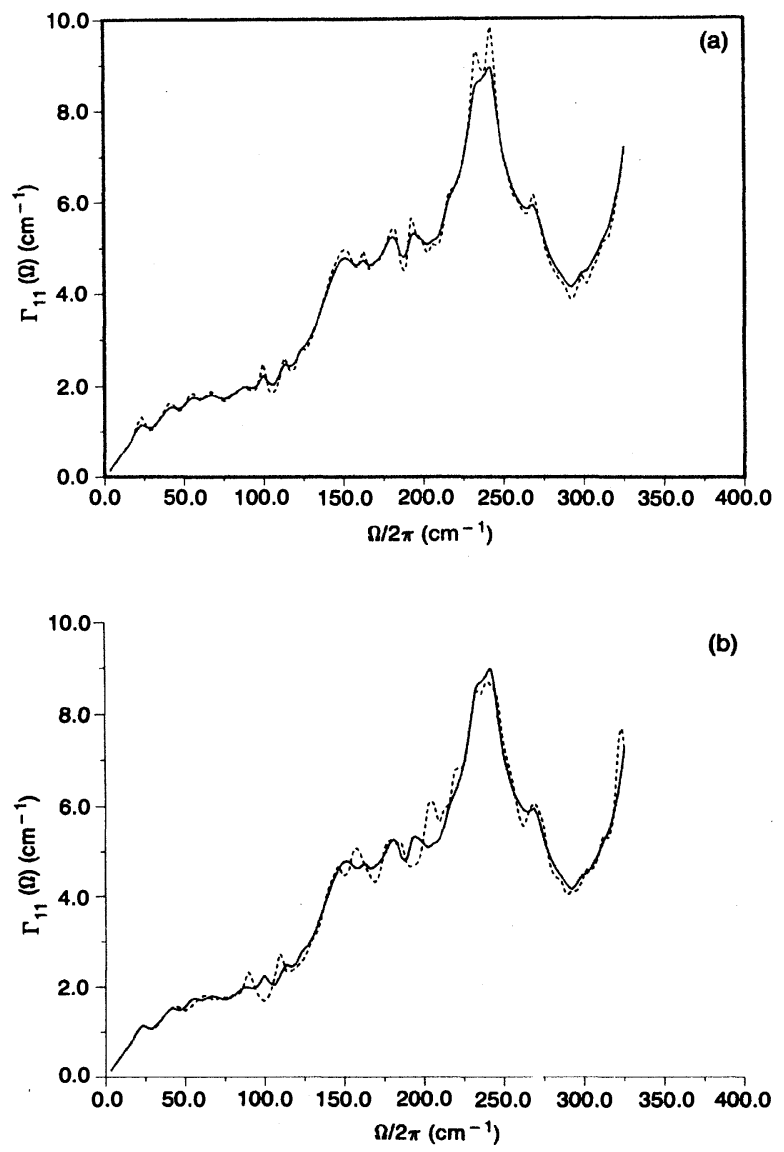

FIG. 5. Effects in $\Gamma_{11}(\Omega)$ of $\epsilon$ and q-space grid size. Results in (a) are based on grid $B$ : Solid and dotted lines correspond, respectively, to $\epsilon=3.33$ and $1.66 \mathrm{~cm}^{-1}$. Results in (b) are based on $\epsilon=3.33 \mathrm{~cm}^{-1}$ : Solid and dotted lines correspond, respectively, to grids $B$ and $A$. In both (a) and (b), $r_{\max } / \sqrt{2} a=1.2$.

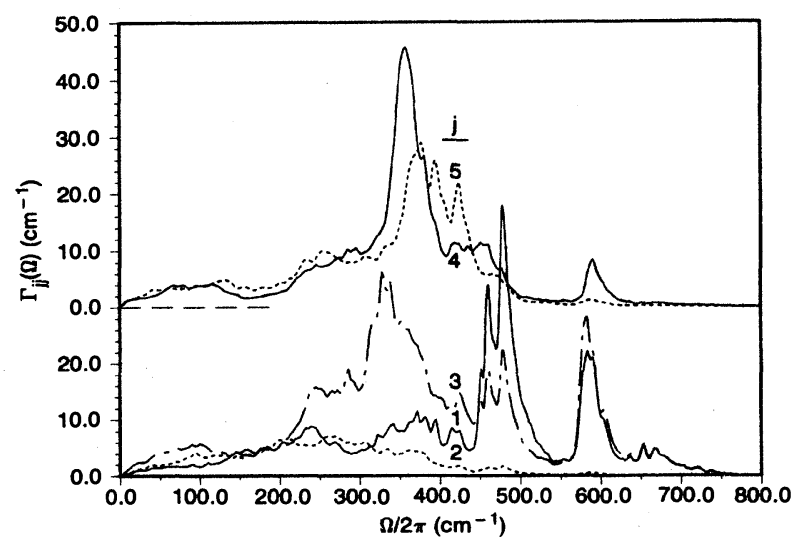

FIG. 6. $\Gamma_{j j}(\Omega)$. 

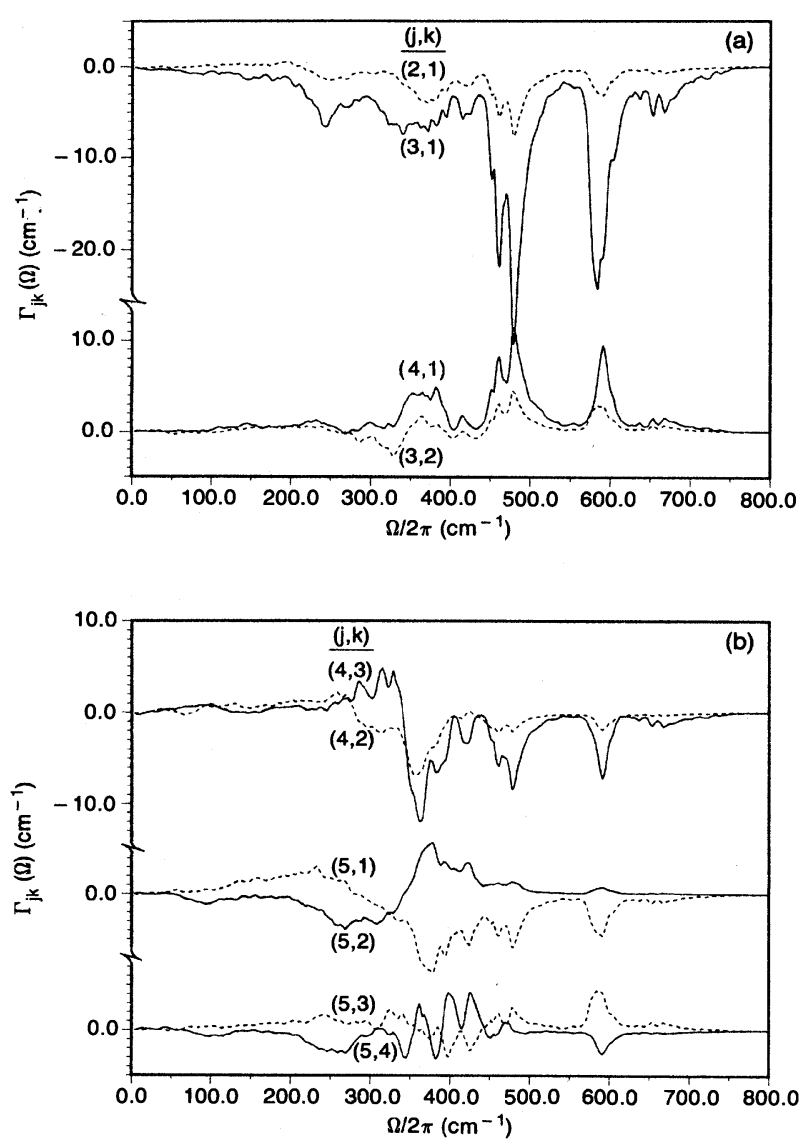

FIG. 7. $\Gamma_{j k}(\Omega), j \neq k$.

(1.2 $\sqrt{2} a$ ) and for grid $A$ (Figs. 6-9). We expect the results for $\Gamma(\Omega)$ to be qualitatively similar to the twophonon density of states (not given), and indeed several of the peaks in $\Gamma(\Omega)$ can be identified with pairs of normalmode branches upon inspecting Fig. 2. Matrix element effects are also evident in the spectra, especially the lack of an observable peak on the scale of the figure near and somewhat above (not shown) a frequency of $800 \mathrm{~cm}^{-1}$. In Table III, we give the diagonal components $\Delta_{j j}^{(3 a)}$ eval-

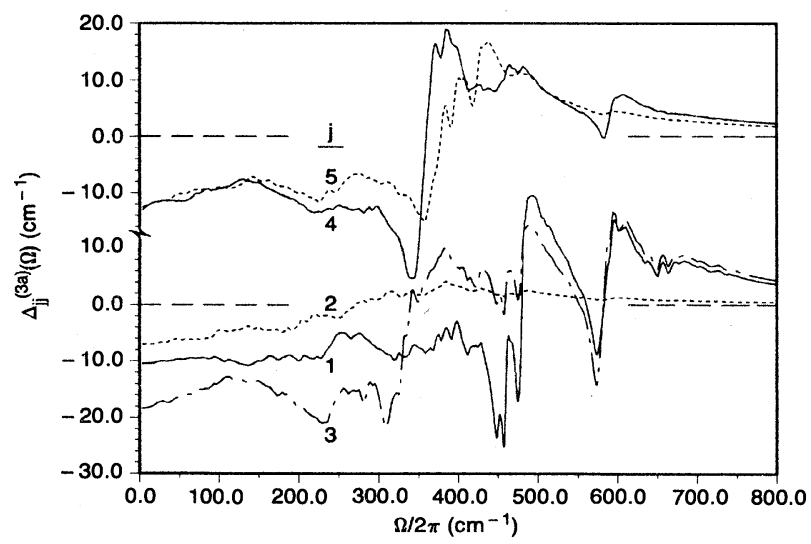

FIG. 8. $\Delta_{j j}^{(3 a)}(\Omega)$.
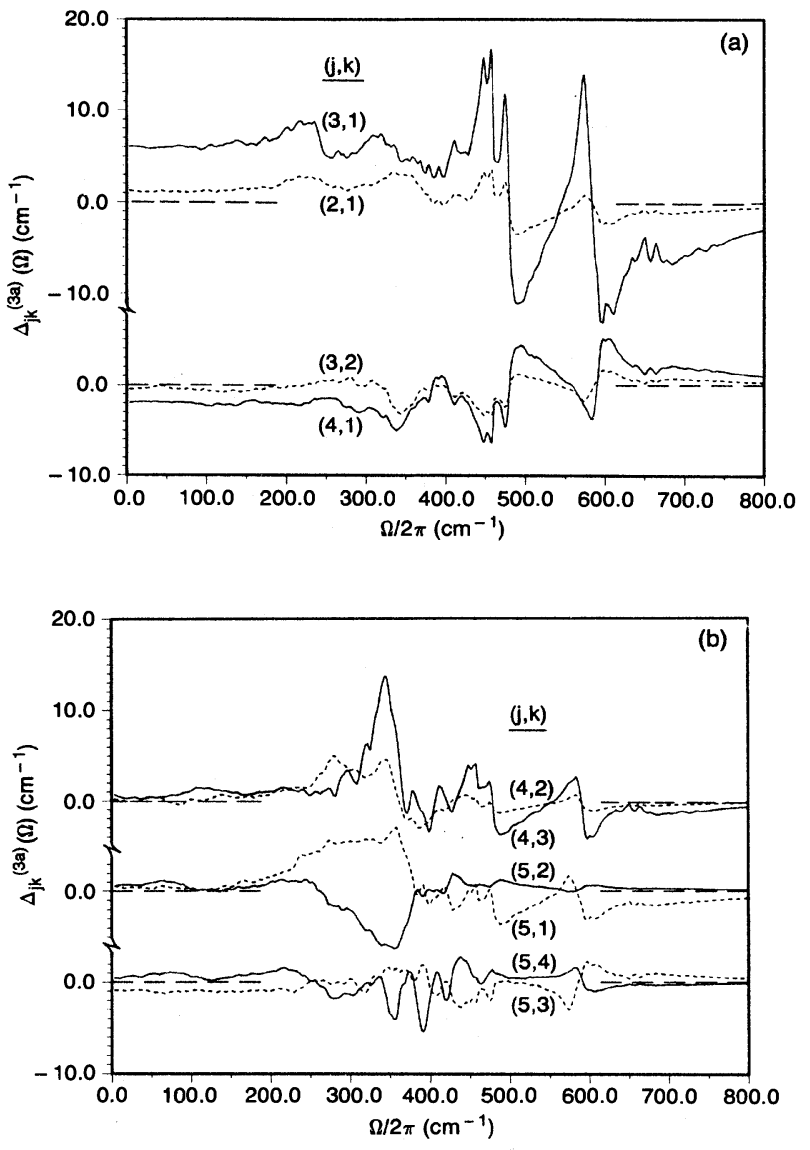

FIG. 9. $\Delta_{j k}^{(3 a)}(\Omega), j \neq k$.

uated at the corresponding harmonic frequencies, and we also include values for the total $\Delta_{j j}$, which incorporate the results of Table I.

\section{RESULTS FOR SUSCEPTIBILITY}

The frequency dependence of the real $\left(\chi_{x x}^{\prime}\right)$ and imaginary $\left(\chi_{x x}^{\prime \prime}\right)$ parts of the lattice dielectric susceptibility is

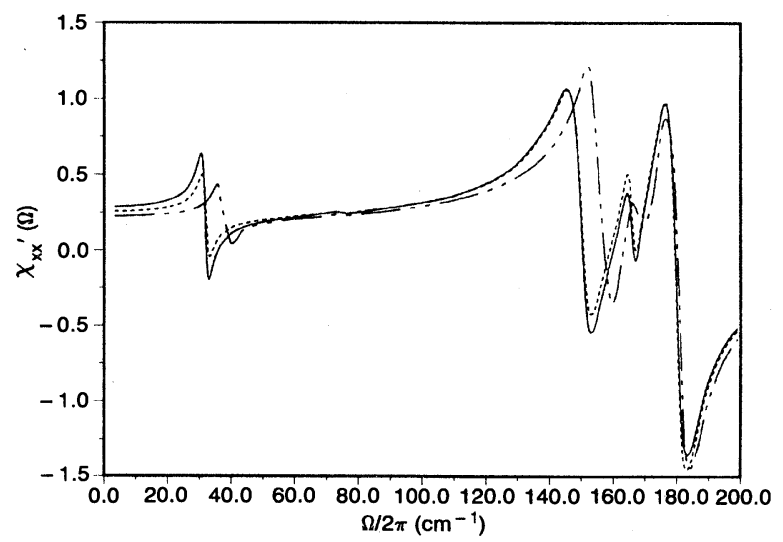

FIG. 10. $\chi_{x x}^{\prime}(\Omega)$. Dotted and broken lines correspond, respectively, to the omission, in the calculation of $\underline{\chi}$, of the offdiagonal elements and of the real part of the self-energy matrix. 


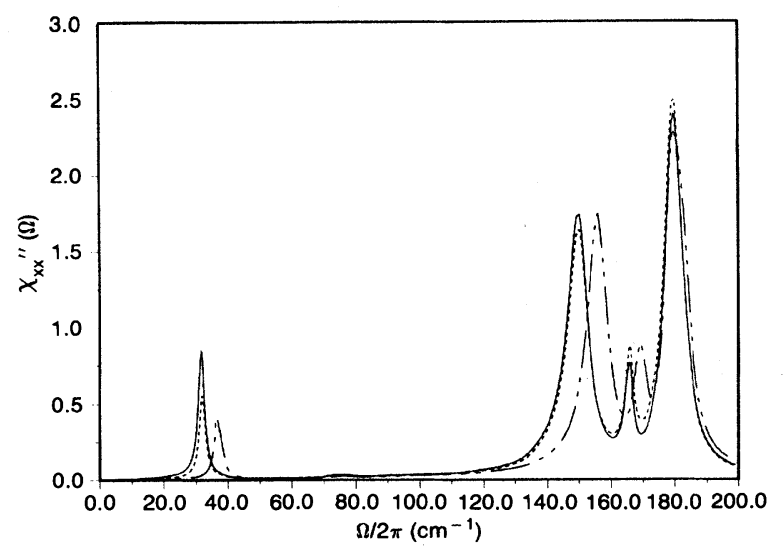

FIG. 11. $\chi_{x x}^{\prime \prime}(\Omega)$. Dotted and broken lines correspond, respectively, to the omission, in the calculation of $\underline{\chi}$, of the offdiagonal elements and of the real part of the self-energy matrix.

shown in Figs. 10 and 11. It is noteworthy that a large off-diagonal Green's-function effect is present in the 30 $\mathrm{cm}^{-1}$ feature in $\chi_{x x}$. (The results correspond to particular values of the numerical parameters, given in the previous section, and we believe them to be sufficiently accurate.) Two effects in the susceptibility of off-diagonal ele-
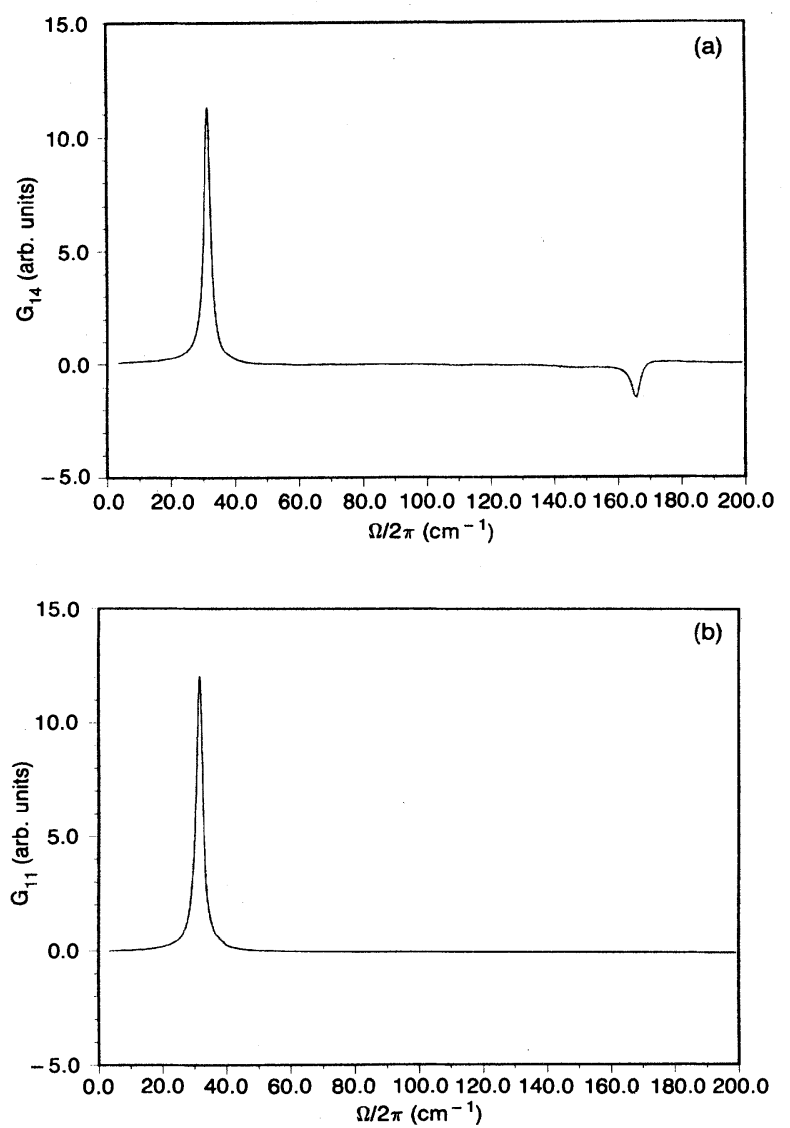

FIG. 12. One-phonon Green's-function components. The imaginary components of (a) an off-diagonal element, and, for comparison, (b) a diagonal element. ments in the self-energy function are possible: One is a shift of peak positions and breadths, which can arise if differences in harmonic levels are of the order of the selfenergy contributions; the other is the contribution to Eq. (1) from the terms involving the off-diagonal components of the Green's function. It is this latter polarization mixing effect which is apparent in our results. As an illustration of the origin of this effect we plot the off-diagonal Green's-function element which couples the lowest-lying mode to one of the other modes in Fig. 12. Although the ratios of the off-diagonal to the diagonal Green's-function elements are proportional to the ratios of self-energies to phonon energies and are therefore small, it is evident from Eq. (1) that if a large disparity exists among the values of $M_{x}$ then a significant off-diagonal effect can arise, which has been pointed out previously through empirical data analyses. ${ }^{10}$

\section{SUMMARY AND CONCLUSIONS}

We made us of the Gordon-Kim $a b$ initio theory (for representing the interionic potential) and anharmonic perturbation theory in order to obtain static equilibrium structural parameters and room-temperature infrared absorption properties of a hypothetical superlattice of alkali halides, particularly $(\mathrm{NaCl})_{2} \mathrm{RbF}$. No additional uncontrolled mathematically simplifying approximations were made. This was done to demonstrate that such calculations are feasible, and because we believe that a study of superlattices of alkali halides, if they could be fabricated, would be of interest for several reasons. In particular (a) they might have some use for their dielectric properties which could be varied by varying the superlattice periodicity or the constituents, (b) they would provide an additional class of materials for testing theory such as that of Gordon and Kim, and (c) they might shed some light on the origin of the supermodulus effect seen in intermetallic superlattices. We have also given our results in great detail because they are the first such results for a superlattice of alkali halides, the first rigorous anharmonic calculations including Coulomb effects without the use of the Ewald method in the cubic anharmonic coefficients and the first anharmonic calculations for which the noncentrosymmetric structural aspect is of some importance.

To summarize our results briefly, a metastable trigonal superlattice structure was obtained although the structure which we had anticipated, based on the rocksalt stacking, was found to be unstable. The structure obtained yielded an infrared-active mode of $30 \mathrm{~cm}^{-1}$-in the far-infrared to submillimeter region-perhaps somewhat lower than could have been expected from zone folding alone because of the unusual structural property of our material, as described in Sec. IV. This mode also contributed significantly to the susceptibility, contrary to the result for a higher-frequency Brillouin-zone-folded mode which gave a much smaller contribution to the susceptibility. In addition, strong peaks in the susceptibility associated with ordinary alkali-halide absorption are apparent in our results. 


\section{ACKNOWLEDGMENTS}

We wish to thank P. B. Allen and R. E. Cohen for helpful conversations. This work was supported by the Office of Naval Research, U.S. Department of Defense.

\section{APPENDIX A: APPLICATION OF GROUP THEORY TO THE ORDINARY CUBIC ANHARMONIC CONTRIBUTION}

Starting with expression (5) of Sec. II we wish to derive an expression for II in terms of a sum over the IBZ rather than the full $\mathrm{BZ}$. In this appendix we shall also rederive certain results of Ref. 15 . We first replace $\mathrm{q}_{1}$ by $\underline{A}(R) \mathrm{q}_{1}$ in the summand and $\Sigma_{\mathrm{q}_{1}}$ by $\Sigma_{\mathrm{q}_{1}}^{\prime} \Sigma_{R}$ where the prime in- dicates a sum over twice the IBZ. ${ }^{27} R$ is a point-group operation, and $\underline{A}(R)$ is the three-dimensional matrix effecting that transformation. Then in each factor of $\Phi$ both of the $\mathrm{q}_{1}$-dependent polarization vectors can be expanded as

$$
\mathrm{e}\left(\kappa, \underline{A}(R) \mathbf{q}_{1}\right)=\underline{A} \mathrm{e}\left(\kappa, \mathrm{q}_{1}\right)
$$

to introduce two factors of $\underline{A}(R)$ into Eq. (5) from each factor of $\Phi$. Three factors of $\underline{A}(R)$ are also introduced into Eq. (5) from each factor of $\Phi$ upon expressing

$$
\Phi_{\alpha \beta \gamma}\left(\begin{array}{lll}
0 & l^{\prime} & l^{\prime \prime} \\
\kappa & \kappa^{\prime} & \kappa^{\prime \prime}
\end{array}\right)
$$

as

$$
A_{\alpha \alpha^{\prime}}(R) A_{\beta \beta^{\prime}}(R) A_{\gamma \gamma^{\prime}}(R) \Phi_{\alpha^{\prime} \beta^{\prime} \gamma^{\prime}}\left(\begin{array}{ccc}
0 & L^{\prime} & L^{\prime \prime} \\
\kappa & \kappa^{\prime} & \kappa^{\prime \prime}
\end{array}\right),
$$

where $\underline{A}\left(R^{-1}\right)\left(\mathbf{X}_{l^{\prime} \kappa^{\prime}}-\mathbf{X}_{0 \kappa}\right) \equiv \mathbf{X}_{L^{\prime} \kappa^{\prime}}-\mathbf{X}_{0 \kappa}$ and $\underline{A}\left(R^{-1}\right)\left(\mathbf{X}_{l^{\prime \prime} \kappa^{\prime \prime}}-\mathbf{X}_{0 \kappa}\right)=\mathbf{X}_{L^{\prime \prime} \kappa^{\prime \prime}}-\mathbf{X}_{0 \kappa^{\prime}}$. Upon making use of the unitary property of $\underline{A}$ and the fact that the vectors $\mathrm{e}(\kappa ; 0, j)$ are basis vectors for irreducible representations of the point group, we obtain

$$
\begin{aligned}
\Phi\left(0, j^{\prime} ; \underline{A}(R) \mathbf{q}_{1}, j_{1} ;-\right. & \left.\underline{A}(R) \mathbf{q}_{1}, j_{2}\right) \\
= & \sum_{\substack{\kappa, \kappa^{\prime}, \kappa^{\prime \prime}, l^{\prime}, l^{\prime \prime}}} \sum_{j^{\prime \prime}} \Phi_{\alpha \beta \gamma}\left[\begin{array}{ccc}
0 & l^{\prime} & l^{\prime \prime} \\
\kappa & \kappa^{\prime} & \kappa^{\prime \prime}
\end{array}\right] e_{\alpha}\left(\kappa ; 0, j^{\prime \prime}\right) e_{\beta}\left(\kappa^{\prime} ; \mathbf{q}_{1}, j_{1}\right) e_{\gamma}\left(\kappa^{\prime \prime} ;-\mathbf{q}_{1}, j_{2}\right) e^{i \mathbf{q}_{1} \cdot\left(\mathbf{x}_{l^{\prime} \kappa^{\prime}}-\mathbf{x}_{\left.l^{\prime \prime} \kappa^{\prime \prime}\right)} D_{j^{\prime \prime} j^{\prime}}\left(R^{-1}\right)\right.}
\end{aligned}
$$

and

$$
\begin{aligned}
\Phi^{*}\left(0, j ; \underline{A}(R) \mathbf{q}_{1}, j_{1} ;-\underline{A}(R) \mathbf{q}_{1}, j_{2}\right) \\
=\sum_{\substack{\kappa, \kappa^{\prime}, \kappa^{\prime \prime}, l^{\prime}, l^{\prime \prime}}} \sum_{j^{\prime \prime \prime}} \Phi_{\alpha \beta \gamma}\left(\begin{array}{lll}
0 & l^{\prime} & l^{\prime \prime} \\
\kappa & \kappa^{\prime} & \kappa^{\prime \prime}
\end{array}\right] e_{\alpha}\left(\kappa ; \mathbf{0}, j^{\prime \prime \prime}\right) e_{\beta}^{*}\left(\kappa^{\prime} ; \mathbf{q}_{1}, j_{1}\right) e_{\gamma}^{*}\left(\kappa^{\prime \prime} ;-\mathbf{q}_{1}, j_{2}\right) e^{-i \mathbf{q}_{1} \cdot\left(\mathbf{X}_{l^{\prime} \kappa^{\prime}}-\mathbf{x}_{l^{\prime \prime} \kappa^{\prime \prime}}\right.} D_{j^{\prime \prime \prime} j}\left(R^{-1}\right)
\end{aligned}
$$

where $\underline{D}$ is a block-diagonal $3 n$-dimensional representation of the point group and is defined by the expressions

$$
\underline{A}\left(R^{-1}\right) \mathbf{e}\left(\kappa ; 0, j_{1}\right)=\sum_{j^{\prime \prime}} D_{j^{\prime \prime} j_{1}}\left(R^{-1}\right) \mathbf{e}\left(\kappa ; 0, j^{\prime \prime}\right) \text {. }
$$

Finally, using the definition of $\Phi\left(\mathbf{q}_{1}, j_{1} ; \mathbf{q}_{2}, j_{2} ; \mathbf{q}_{3}, j_{3}\right)$ and combining the above two expressions we may write

$$
\Pi_{j j^{\prime}}^{(3 a)}=\sum_{\mathbf{q}_{1}}^{\prime} \sum_{j_{1}, j_{2}} F^{(3 a)}(\Omega, \omega) \sum_{j^{\prime \prime}, j^{\prime \prime \prime}} \sum_{R} D_{j^{\prime \prime \prime} j}\left(R^{-1}\right) D_{j^{\prime \prime} j^{\prime}}\left(R^{-1}\right) \Phi^{*}\left(\mathbf{0}, j^{\prime \prime \prime} ; \mathbf{q}_{1}, j_{1} ;-\mathbf{q}_{1}, j_{2}\right) \Phi\left(0, j^{\prime \prime} ; \mathbf{q}_{1}, j_{1} ;-\mathbf{q}_{1}, j_{2}\right) \text {. }
$$

Expressing the mode indices in terms of those corresponding to irreducible representations we replace $j$ by $(t, n, \mu)$ where $t$ and $\mu$ denote the basis partner and the irreducible representation, respectively, and $n$ denotes the order of the appearance of that representation, say, according to frequency. Thus, we rewrite the above expression as

$$
\begin{aligned}
\Pi_{(t, n, \mu),(r, k, v)}^{(3 a)}=\sum_{\mathbf{q}_{1}} \sum_{j_{1}, j_{2}} F^{(3 a)}(\Omega, \omega) \sum_{s} \sum_{u} \sum_{R} \mathscr{D}_{u t}^{\mu}\left(R^{-1}\right) D_{s r}^{v}\left(R^{-1}\right) \\
\\
\quad \times \Phi^{*}\left(0,(u, n, \mu) ; \mathbf{q}_{1}, j_{1} ;-\mathbf{q}_{1}, j_{2}\right) \Phi\left(0,(s, k, v) ; \mathbf{q}_{1}, j_{1} ;-\mathbf{q}_{1}, j_{2}\right),
\end{aligned}
$$

where the $\mathscr{D}^{\mu}(R)$ matrices form the $\mu$ th irreducible representation, and where it is presumed that the polarization vectors are chosen such that those corresponding to different values of $n$ but the same $\mu$ yield identical contributions to $\underline{D}$. The latter is a necessary consideration because in practice those equivalent contributions to $\underline{D}$ could be related by a similarity transformation. Employing the orthogonality theorem for irreducible representation matrices ${ }^{28}$ we finally obtain

$$
\Pi_{(t, n, \mu),(r, k, v)}^{(3 a)}=\sum_{\mathbf{q}_{1}}^{\prime} \sum_{j_{1}, j_{2}} F^{(3 a)}(\Omega, \omega) \frac{g}{d_{\mu}} \delta_{\mu \nu} \delta_{t r} \sum_{s} \Phi^{*}\left(0,(s, n, \mu) ; \mathbf{q}_{1}, j_{1} ;-\mathbf{q}_{1}, j_{2}\right) \Phi\left(0,(s, k, v) ; \mathbf{q}_{1}, j_{1} ;-\mathbf{q}_{1}, j_{2}\right)
$$


where $g$ is the order of the point group, e.g., 6 in our case and $d_{\mu}$ is the dimensionality of the $\mu$ th irreducible representation. The implications of this result are that degeneracies are not broken by anharmonic effects, expressed by the $\delta_{t r}$ dependence, and that one may perform the $q_{1}$ summation of the original expression for $\pi^{(3 a)}$ over the IBZ, provided that the results are averaged over appropriate degenerate modes or pairs of degenerate modes in the case of diagonal and off-diagonal components, respectively. Furthermore, we wish to stress that the essential feature of this proof was that the zone-center polarization vectors transformed as partner basis functions of the irreducible representations of the point group. In the case of the rocksalt structure for example the optical zone-center modes transform as partners of a threedimensional irreducible representation of the cubic point group despite the fact that the triple degeneracy of the modes is broken by the macroscopic electric field. Hence, with some care it is possible to restrict the $q$ sum to the IBZ in that case as well.

\section{APPENDIX B: APPLICATION OF GROUP THEORY TO THE INSTANTANEOUS PHONON CUBIC ANHARMONIC CONTRIBUTION}

We consider the effects of the $C_{3 v}$ point-group symmetry on expression 8 . We replace $\mathrm{q}_{2}$ by $\underline{A}(R) \mathrm{q}_{2}$ and rewrite the $\mathbf{q}_{2}$ summation as $\Sigma_{\mathbf{q}_{2}}^{\prime} \Sigma_{R}$, where the prime denotes a sum over twice the IBZ. ${ }^{27}$ Using the fact that $\omega(\underline{A} \mathbf{q}, j)=\omega(\mathbf{q}, j)$ we next carry out the $R$ summation over relevant terms in Eq. (8). In particular, we need to evaluate

$$
\begin{aligned}
Y & \equiv \sum_{R} \Phi\left(0, j_{1} ; \underline{A}(R) \mathbf{q}_{2}, j_{2} ;-\underline{A}(R) \mathbf{q}_{2}, j_{2}\right) \\
& =\sum_{R} \sum_{\substack{\kappa, \kappa^{\prime}, \kappa^{\prime \prime}, l^{\prime}, l^{\prime \prime}}} \Phi_{\alpha \beta \gamma}\left[\begin{array}{ccc}
0 & l^{\prime} & l^{\prime \prime} \\
\kappa & \kappa^{\prime} & \kappa^{\prime \prime}
\end{array}\right] e_{\alpha}\left(\kappa ; 0, j_{1}\right) e_{\beta}\left(\kappa^{\prime} ; \underline{A}(R) \mathbf{q}_{2}, j_{2}\right) e_{\gamma}\left(\kappa^{\prime \prime} ;-\underline{A}(R) \mathbf{q}_{2}, j_{2}\right) e^{i \underline{A}(R) \mathbf{q}_{2} \cdot\left(\mathbf{x}_{l^{\prime} \kappa^{\prime}}-\mathbf{X}_{l^{\prime \prime} \kappa^{\prime \prime}}\right)} .
\end{aligned}
$$

Using the unitarity of $\underline{A}(R)$ and the transformation properties, e( $\kappa ; \underline{A}(R) \mathbf{q}, j)=\underline{A}(R) \mathbf{e}(\kappa ; \mathbf{q}, j)$ and

$$
\Phi_{\alpha \beta \gamma}\left(\begin{array}{ccc}
0 & l^{\prime} & l^{\prime \prime} \\
\kappa & \kappa^{\prime} & \kappa^{\prime \prime}
\end{array}\right]=A_{\alpha \alpha^{\prime}}(R) A_{\beta \beta^{\prime}}(R) A_{\gamma \gamma^{\prime}}(R) \Phi_{\alpha^{\prime} \beta^{\prime} \gamma^{\prime}}\left(\begin{array}{lll}
0 & L^{\prime} & L^{\prime \prime} \\
\kappa & \kappa^{\prime} & \kappa^{\prime \prime}
\end{array}\right],
$$

where lattice sites $L^{\prime}$ and $L^{\prime \prime}$ are defined as in Appendix A, one can show that

$$
Y=\sum_{R} \sum_{\substack{\kappa, \kappa^{\prime}, \kappa^{\prime \prime}, l^{\prime}, l^{\prime \prime}}} \Phi_{\alpha^{\prime} \beta^{\prime} \gamma^{\prime}}\left(\begin{array}{ccc}
0 & l^{\prime} & l^{\prime \prime} \\
\kappa & \kappa^{\prime} & \kappa^{\prime \prime}
\end{array}\right) A_{\alpha^{\prime} \alpha}\left(R^{-1}\right) e_{\alpha}\left(\kappa ; 0, j_{1}\right) e_{\beta^{\prime}}\left(\kappa^{\prime} ; \mathbf{q}_{2}, j_{2}\right) e_{\gamma^{\prime}}\left(\kappa^{\prime \prime} ;-\mathbf{q}_{2}, j_{2}\right) e^{i \mathbf{q}_{2} \cdot\left(\mathbf{X}_{l^{\prime} \kappa^{\prime}}-\mathbf{X}_{l^{\prime \prime} \kappa^{\prime \prime}}\right)}
$$

The quantity $A_{\alpha^{\prime} \alpha}\left(R^{-1}\right) e_{\alpha}\left(\kappa ; 0, j_{1}\right)$ can be written in terms of the irreducible representations of the point group only if we define the internal-line zone-center modes in Fig. 1(b) to correspond to polarization vectors that are basis vectors of the irreducible representations of the point group. Making that definition, which indeed is justifiable from the discussion in the text we obtain

$$
Y=\sum_{\substack{\kappa, \kappa^{\prime}, \kappa^{\prime \prime}, l^{\prime}, l^{\prime \prime}}} \sum_{j^{\prime \prime}} D_{j^{\prime \prime} j_{1}}(R) \Phi_{\alpha \beta \gamma}\left(\begin{array}{ccc}
0 & l^{\prime} & l^{\prime \prime} \\
\kappa & \kappa^{\prime} & \kappa^{\prime \prime}
\end{array}\right] e_{\alpha}\left(\kappa ; 0, j^{\prime \prime}\right) e_{\beta}\left(\kappa^{\prime} ; \mathbf{q}_{2}, j_{2}\right) e_{\gamma}\left(\kappa^{\prime \prime} ;-\mathbf{q}_{2}, j_{2}\right) e^{i \mathbf{q}_{2} \cdot\left(\mathbf{X}_{l^{\prime} \kappa^{\prime}}-\mathbf{X}_{l^{\prime \prime} \kappa^{\prime \prime}}\right)}
$$

We consider the six point-group operations of $C_{3 v}$ and three irreducible representations. ${ }^{28,29}$ The only nonzero contribution to the $R$ summation is seen to arise from the identity representation, which corresponds to modes with trigonal axis polarization. For $j_{1}$ corresponding to any one of these LO (see text) modes we obtain

$$
\sum_{R} D_{j^{\prime \prime} j_{1}}\left(R^{-1}\right)=6 \delta_{j_{1} j^{\prime \prime}}
$$

Thus

$$
Y=6 \sum_{\substack{\kappa, \kappa^{\prime}, \kappa^{\prime \prime}, l^{\prime}, l^{\prime \prime}}} \Phi_{\alpha \beta \gamma}\left[\begin{array}{lll}
0 & l^{\prime} & l^{\prime \prime} \\
\kappa & \kappa^{\prime} & \kappa^{\prime \prime}
\end{array}\right] e_{\alpha}\left(\kappa ; 0, j_{1}\right) e_{\beta}\left(\kappa^{\prime} ; \mathbf{q}_{2}, j_{2}\right) e_{\gamma}\left(\kappa^{\prime \prime} ;-\mathbf{q}_{2}, j_{2}\right) e^{i \mathbf{q}_{2} \cdot\left(\mathbf{X}_{l^{\prime} \kappa^{\prime}}-\mathbf{X}_{l^{\prime \prime} \kappa^{\prime \prime}}\right)}
$$


which signifies that Eq. (8) may be evaluated by restricting the $\mathbf{q}_{2}$ summation to the IBZ and the $j_{1}$ summation to LO modes for $\mathbf{q} \rightarrow \mathbf{0}$ along the trigonal axis. That $j_{1}$ corresponds to the identity representation can be used to prove the fact that $j$ and $j^{\prime}$ must, for nonzero $\Pi_{j j^{\prime}}$, correspond to the same row of the same irreducible representation: On the other hand we could have used this more generally derived fact ${ }^{15}$ to obtain that $j_{1}$ corresponds to the identity representation.

In our numerical evaluation we simply employed Eqs.
(8) and (9) except for the fact that the $\mathbf{q}_{2}$ sum was performed over the IBZ. [Of course, in Eq. (9), $N$ is essentially the number of points in the grid-appropriate fractional weighing is given for points on the boundary of the IBZ.] Hence, no further possible reduction of Eq. (8) will be discussed. We caution that in the numerical evaluation of Eq. (8) it is important to insure that the polarization vectors of modes corresponding to the same irreducible representation, but to different frequencies, yield identical portions of the $\underline{D}$ matrix.
${ }^{1}$ D. Wolf and J. F. Lutsko, Phys. Rev. Lett. 60, 1170 (1988).

2J. R. Hardy and A. M. Karo, Phys. Rev. B 26, 3327 (1982).

${ }^{3}$ J. R. Hardy, P. J. Edwardson, and J. A. O'Gara, Phys. Rev. B 30, 6136 (1984).

${ }^{4}$ R. G. Gordon and Y. S. Kim, J. Chem. Phys. 56, 3122 (1972).

${ }^{5}$ R. E. Cohen, L. L. Boyer, and M. J. Mehl, Phys. Rev. B 35, 5749 (1987).

${ }^{6} \mathrm{H}$. Bilz, D. Strauch, and R. K. Wehner, in Handbuch der Physik, edited by S. Flugge and L. Genzel (Springer-Verlag, New York, 1984), Vol. XXV/2d, p. 1.

${ }^{7}$ U. Knohl, Phys. Status Solidi B 53, 295 (1972).

${ }^{8}$ E. R. Cowley, G. Jacucci, M. L. Klein, and I. R. McDonald, Phys. Rev. B 14, 1758 (1976).

${ }^{9}$ Z. Gong, G. K. Horton, and E. R. Cowley, Phys. Rev. B 38, 10820 (1989).

${ }^{10}$ A. A. Maradudin and A. E. Fein, Phys. Rev. 128, 2589 (1962).

${ }^{11}$ P. B. Allen (private communication).

${ }^{12}$ A. S. Barker, Jr. and J. J. Hopfield, Phys. Rev. B 5, 1732 (1964).

${ }^{13}$ R. A. Cowley, Philos. Mag. 11, 673 (1965).

${ }^{14}$ E. Haro, M. Balkanski, R. F. Wallis, and K. H. Wanser, Phys. Rev. B 34, 5358 (1986).

${ }^{15}$ A. A. Maradudin and I. P. Ipatova, J. Math. Phys. 9, 525 (1968).

${ }^{16}$ J. L. Birman, Theory of Crystal Space Groups and Lattice Dynamics-Infra-Red and Raman Optical Processes of Insulating Crystals (Springer-Verlag, Berlin, 1984).
${ }^{17}$ R. F. Wallis, I. P. Ipatova, and A. A. Maradudin, Fiz. Tverd. Tela (Leningrad) 8, 1064 (1966) [Sov. Phys.-Solid State 8, 850 (1966)].

${ }^{18}$ R. A. Cowley, Adv. Phys. 12, 421 (1963).

${ }^{19}$ A. A. Maradudin and R. F. Wallis, Phys. Rev. 123, 777 (1961); R. F. Wallis and A. A. Maradudin, ibid. 125, 1277 (1962).

${ }^{20}$ V. S. Vinogradov, Fiz. Tverd. Tela (Leningrad) 4, 712 (1962) [Sov. Phys.-Solid State 4, 519 (1962)].

${ }^{21}$ R. Loudon, Adv. Phys. 13, 423 (1964).

${ }^{22}$ G. Leibfried and W. Ludwig, in Solid State Physics, edited by F. Seitz and D. Turnbull (Academic, New York, 1961), Vol. 12, p. 276.

${ }^{23}$ M. Parinello and A. Rahman, Phys. Rev. Lett. 45, 1196 (1980).

${ }^{24}$ L. L. Boyer, Phys. Rev. B 23, 3675 (1981).

${ }^{25}$ E. Burstein, in Lattice Dynamics, edited by R. F. Wallis (Pergamon, Oxford, 1965), p. 315.

${ }^{26}$ We write

$$
\delta(x)=\frac{2 \epsilon}{x^{2}+(2 \pi \epsilon)^{2}}, \frac{1}{(x)_{\mathbf{P}}}=\frac{x}{x^{2}+(2 \pi \epsilon)^{2}} .
$$

${ }^{27}$ As defined here the IBZ accounts for time-reversal symmetry in addition to space-group symmetry.

${ }^{28}$ J. F. Cornwell, Selected Topics in Solid State Physics $X$, edited by E. P. Wohlfarth (North-Holland, Amsterdam, 1969).

${ }^{29}$ Only two irreducible representations enter $\underline{D}$, however. 